\title{
Apalutamide - 10 clinically important facts
}

\section{Kajetan Juszczak ${ }^{1,2}$, Tomasz Drewa ${ }^{1,3}$}

'Department of Urology and Andrology, Collegium Medicum, Nicolaus Copernicus University, Bydgoszcz, Poland

${ }^{2}$ Department of Urology Memorial Rydygier Hospital, Cracow, Poland

${ }^{3}$ Department of General and Oncological Urology, Nicolaus Copernicus Hospital, Torun, Poland

\section{Correspondence:}

Kajetan Juszczak

Department of Urology and Andrology, Collegium Medicum, Nicolaus Copernicus

University, Bydgoszcz, Poland 85-094 Bydgoszcz, ul. Marii

Skłodowskiej-Curie 9

e-mail:kaj.juszczak@gmail.com

Received:

12.09.2021

Accepted:

30.09.2021

DOI: 10.24292/01.OR.123300921.2

Copyright $\odot$ Medical Education.

All rights reserved.

\section{ABSTRACT}

Apalutamide belongs to the ARTA group. This drug is a new generation selective androgen receptor inhibitor. Patients with prostate cancer develop resistance to castration testosterone levels over time during hormone therapy. Therefore, it is necessary to consider additional therapeutic options based on drugs from the ARTA group. Apalutamide is used in the treatment of patients with non-metastatic as well as metastatic prostate cancer. This article presents the most important clinical facts about apalutamide.

Key words: apalutamide, prostate cancer, nonmetastatic, metastatic, CRPC, CSPC 


\section{INTRODUCTION}

Patients with castration-resistant nonmetastatic prostate cancer (nmCRPC) are identified by increasing levels of prostate-specific antigen (PSA) and the absence of distant metastases in conventional imaging in the context of androgen deprivation therapy (ADT) [1]. In the absence of treatment modification, most patients with nmCRPC disease progress to the stage of metastatic disease $[2,3]$. The previous observations have shown that in patients with $\mathrm{nmCRPC}$ prostate cancer, a shorter PSA doubling time (PSADT) is strongly associated with a higher risk of distant metastases and death $[4,5]$. It should be remembered that the overarching goals of the treatment of patients with nmCRPC prostate cancer include the prevention of distant metastases, the maintenance of quality of life at the optimal level for a given patient, as well as the extension of overall survival (OS) [6]. Therefore, in the case of PSA marker progression in patients with nmCRPC pros tate cancer on conventional ADT therapy (using agonists or antagonists of gonadotropin releasing hormone $\mathrm{Gn}-\mathrm{RH}$ ), it is worth considering the use of modern anti-androgen ARTA (androgen receptor targeted agents) drugs (e.g. apalutamide) in order to optimize hormone therapy and delay the progression of the neoplastic disease.

The aim of the publication is to present the 10 most important clinical facts about the modern anti-androgen drug, i.e. apalutamide, which will allow us to better understand the current position of this drug in the treatment of patients with prostate cancer.

The results of the SPARTAN and TITAN studies provide many important facts. The SPARTAN study is a multicentre, double-blind (phase III) clinical trial in which 1,207 patients with nmCRPC prostate cancer (with PSADT less than 10 months of age) were randomized $(2: 1)$ to receive apalutamide $240 \mathrm{mg}$ orally once daily in combination with pharmacological or surgical castration (ADT) $(n=806)$ or placebo once daily with ADT $(n=401)$. The median follow-up in the SPARTAN study was 50.4 months. The median duration of apalutamide and placebo treatment was 32.9 and 11.5 months, respectively [7]. The TITAN study, on the other hand, is a multicentre, double-blind (phase III) clinical trial that assessed the efficacy and safety of apalutamide in combination with androgen deprivation (ADT + apalutamide) compared to placebo combined with androgen deprivation (ADT + placebo) among 1,052 patients with metastatic, hormone-sensitive prostate cancer (mCRPC).
FACT 1. APALUTAMIDE IS A MODERN ANTI-

\section{-ANDROGEN DRUG}

Apalutamide is a selective androgen receptor (AR) inhibitor which binds directly to the ligand binding domain of the $A R$. Apalutamide prevents translocation of the androgen receptor complex into the nucleus, inhibits DNA binding, inhibits AR-dependent transcription, and has no androgen receptor agonist activity. Apalutamide reduces the proliferation of neoplastic cells and increases apoptosis, leading to a strong anti-neoplastic activity [8]. In addition, apalutamide binds poorly to GABA receptors (IC50 $=3 \mu \mathrm{M})$ and its concentrations in the central nervous system are 4 times lower than that of enzalutamide, indicating a potentially lower risk of seizures compared to enzalutamide. Additionally, unlike the first generation antiandrogens (e.g. bicalutamide), apalutamide selectively and irreversibly binds to AR with high affinity, but binds only minimally to other hormone and neurotransmitter receptors.

\section{FACT 2. APALUTAMIDE AND OVERALL SURVIVAL} IN A PATIENT WITH NMCRPC PROSTATE CANCER

The use of apalutamide therapy in patients undergoing androgen deprivation (ADT) increases overall survival compared to placebo in patients with nmCRPC. In the phase III SPARTAN trial, there was a $22 \%$ reduction in the risk of death in the apalutamide-treated group despite a 19\% treatment change rate (ADT + placebo ADT + apalutamide) and a higher rate of post-treatment in the placebo group. The median overall survival was significantly longer for the apalutamide treated group compared to the placebo group, reaching a predetermined statistical significance (73.9 vs. 59.9 months, hazard ratio: 0.78 (95\% confidence interval: 0.64-0.96); $p=0.016$ ) [7]. Of the 428 deaths, 274 (out of 806 patients) occurred in the apalutamide group and 154 (out of 401 patients) in the placebo group.

\section{FACT 3. APALUTAMIDE AND PSA MARKER PROGRESSION IN A PATIENT WITH NMCRPC PROSTATE} CANCER

A total of 572 patients with nmCRPC prostate cancer experienced PSA marker progression: 235 of 806 patients received apalutamide and 337 of 401 patients received placebo. The median time to PSA marker progression for the apalutamide and placebo groups was 40.5 and 3.7 months, respectively. Apalutamide reduced the risk of PSA marker progression by $93 \%$ compared to placebo. Hazard ratio: 0.07 (95\% confidence interval: 0.06-0.09); $\mathrm{p}<0.0001)$. 
FACT 4. APALUTAMIDE AND METASTASIS-FREE SURVIVAL IN A PATIENT WITH nmCRPC PROSTATE CANCER

In the SPARTAN study, the end-point metastatis-free survival (MFS) analysis was performed after the onset of distant metastases or death in 378 patients ( $23 \%$ in the apalutamide group and $48 \%$ in the placebo group). The median MFS was 40.5 months in the apalutamide group and 16.2 months in the placebo group. Among patients who developed metastases, the majority of metastases was the skeletal system (in $60.5 \%$ of patients in the group treated with apalutamide and $54.4 \%$ in the placebo group) [9].

\section{FACT 5. APALUTAMIDE AND PROGRESSION-FREE} SURVIVAL IN A PATIENT WITH nmCRPC PROSTATE

\section{CANCER}

In patients with nmCRPC prostate cancer, a $71 \%$ reduction in the risk of local progression, distant progression, or death was observed during apalutamide therapy. Median progression-free survival was 40.5 and 14.7 months for apalutamide and placebo, respectively. Hazard ratio: 0.29 (95\% confidence interval: $0.24-0.36) ; p<0.001[9]$.

\section{FACT 6. APALUTAMIDE REDUCES THE PROPORTION} OF PATIENTS WITH PROGRESSION OF SYMPTOMS IN

\section{A PATIENT WITH nMCRPC PROSTATE CANCER}

In patients with nmCRPC prostate cancer, a $55 \%$ reduction in risk of skeletal-related events (SREs), pain progression/symptom worsening or clinically relevant symptoms was reported during apalutamide therapy. 264 patients experienced symptomatic progression, 156 of 806 in the apalutamide group and 108 of 401 in the placebo group. An updated analysis confirmed the observed benefit of apalutamide treatment to reduce the risk of symptomatic progression compared to placebo. Hazard ratio: 0.57 (95\% confidence interval: $0.44-0.73$ ); $p<0.001$ (the median was not reached in any of the groups) $[7,9]$.

\section{FACT 7. APALUTAMIDE REDUCES THE RISK OF} INITIATING CHEMOTHERAPY IN A PATIENT WITH nmCRPC PROSTATE CANCER

The results of the SPARTAN study showed that 258 patients with prostate cancer started chemotherapy due to disease progression in the apalutamide group (155/806 patients) and the placebo group (103/401 patients). Treatment with apalutamide reduced the risk of initiating cytotoxic chemotherapy by
$37 \%$ compared with the placebo group. Hazard ratio: 0.63 (95\% confidence interval: $0.49-0.81$ ); $p=0.0002$ (the median was not reached in either group) [7].

\section{FACT 8. APALUTAMIDE HAS A GOOD TOLERANCE PROFILE}

The overall incidence of any adverse reactions (AEs) was similar between the apalutamide and placebo treated groups. Adverse reactions (all grades) were observed in $97 \%$ of apalutamide patients, $94 \%$ of placebo patients and $90 \%$ of the cross group patients. The severe AE rates (SAE) adjusted for exposure per 100 patient-years were 13.7 in the apalutamide group and 22.2 in the placebo group. No AE or SAE rates had a noticeable increase with the duration of apalutamide and placebo use [7].

\section{FACT 9. SIDE EFFECTS OF APALUTAMIDE}

The most common side effects during apalutamide therapy are fatigue $(26 \%)$, rash ( $26 \%$ of each grade and $6 \%$ of grade 3 or 4 ), hypertension (22\%), hot flushes (18\%), arthralgia (17\%), diarrhea (16\%), falls (13\%) and weight loss (13\%). Moreover, other important side effects include fractures (11\%) and hypothyroidism (8\%) [10].

\section{FACT 10. APALUTAMIDE IS USED IN A PATIENT WITH HORMONE SENSITIVE METASTATIC PROSTATE CANCER} After nearly 4 years of follow-up, a final analysis of the TITAN clinical trial showed that in a broad population of patients with metastatic prostate cancer (mCSPC), combination therapy (apalutamide + ADT) improves overall survival with a 35\% reduction in the risk of death. In addition, a $48 \%$ reduction in the risk of death was observed in the switch group (ADT + placebo $\rightarrow$ ADT + apalutamide). Additionally, there have been continued benefits with apalutamide in delaying to testosterone castration resistance (CSPC $\rightarrow$ CRPC). The quality of life was maintained with an acceptable safety profile [11].

\section{CONCLUSION}

The presented clinical facts indicate that in patients with prostate cancer, both in the non-metastatic stage of CRPC and in the metastatic stage of CSPC, the use of an effective modern hormone therapy based on apalutamide in combination with androgen deprivation brings a measurable benefit to patients, significantly prolonging survival and maintaining its quality. 


\section{References}

1. Mateo J, Fizazi K, Gillessen S et al. Managing nonmetastatic castration-resistant prostate cancer. Eur Urol. 2019; 75(2): 285-93.

2. Dai C, Heemers H, Sharifi N. Androgen signaling in prostate cancer. Cold Spring Harb Perspect Med. $2017 ;$ 7(9): a030452.

3. Sharifi N, Gulley JL, Dahut WL. An update on androgen deprivation therapy for prostate cancer. Endocr Relat Cancer. 2010; $17(4)$ : R305315.

4. Smith MR, Kabbinavar F, Saad F et al. Natural history of rising serum prostate-specific antigen in men with castrate nonmetastatic prostate cancer. J Clin Oncol. 2005; 23(13): 2918-25.

5. Whitney CA, Howard LE, Freedland SJ et al. Impact of age, comorbidity, and PSA doubling time on long-term competing risks for mortality among men with non-metastatic castration-resistant prostate cancer. Prostate Cancer Prostatic Dis. 2019; 22: 252-60.

6. Saad F, Cella D, Basch E et al. Effect of apalutamide on health-related quality of life in patients with non-metastatic castration-resistant prostate cancer: an analysis of the SPARTAN randomised, placebo-controlled, phase 3 trial. Lancet Oncol. 2018; 19: 1404-16.

7. Smith MR, Saad F, Chowdhury S et al. Apalutamide and Overall Survival in Prostate Cancer. Eur Urol. 2021; 79(1): 150-8.

8. Clegg NJ, Wongvipat J, Joseph JD et al. ARN-509: a novel antiandrogen for prostate cancer treatment. Cancer Res. 2012 ; 72(6): $1494-503$.

9. Smith MR, Saad F, Chowdhury S et al; SPARTAN Investigators. Apalutamide Treatment and Metastasis-free Survival in Prostate Cancer. N Engl J Med. 2018; 378(15): 1408-18.

10. Charakterystyka produktu leczniczego ERLEADA ${ }^{\oplus}$ (apalutamid). Luty 2021.

11. Chi KN, Agarwal N, Bjartell A et al; TITAN Investigators. Apalutamide for Metastatic, Castration-Sensitive Prostate Cancer. N Engl J Med. 2019; 381(1): 13-24. 